\title{
A Single Nucleotide Polymorphism in SLC7A5 Was Associated With Clinical Response in Multiple Myeloma Patients
}

\author{
MING J. POI ${ }^{1,2,3}$, JUNAN LI ${ }^{1,4}$, JASMINE A. JOHNSON ${ }^{1 \#}$, YU KYOUNG CHO ${ }^{3}$, \\ DOUGLAS W. SBOROV ${ }^{5}$, MITCH A. PHELPS ${ }^{3,4}$ and CRAIG C. HOFMEISTER ${ }^{6}$ \\ ${ }^{1}$ Division of Pharmacy Practice and Science, College of Pharmacy, \\ The Ohio State University, Columbus, OH, U.S.A.; \\ ${ }^{2}$ Department of Pharmacy, The Ohio State University Wexner Medical Center, Columbus, OH, U.S.A.; \\ ${ }^{3}$ Division of Pharmaceutics and Pharmaceutical Chemistry, \\ College of Pharmacy, The Ohio State University, Columbus, OH, U.S.A.; \\ ${ }^{4}$ Comprehensive Cancer Center, The Ohio State University, Columbus, OH, U.S.A.; \\ ${ }^{5}$ Division of Hematology and Hematologic Malignancies, \\ University of Utah - Huntsman Cancer Institute, Salt Lake City, UT, U.S.A.; \\ ${ }^{6}$ Deparment of Hematology \& Oncology, Winship Cancer Institute of Emory University, Atlanta, GA, U.S.A.
}

\begin{abstract}
Background/Aim: SLC7A5 is recognized as the major mediator of melphalan uptake into multiple myeloma (MM) cells; however, its contribution to the inter-patient variability of melphalan efficacy and toxicity is yet to be well elucidated. This study aimed to investigate the impact of a single nucleotide polymorphism (SNP) rs4240803 in SLC7A5 on the gene expression, ex vivo sensitivity to melphalan, and clinical outcomes in MM patients who were undergoing autologous stem cell transplantation with high-dose melphalan. Materials and Methods: Peripheral blood mononuclear cells (PBMC) were collected from $108 \mathrm{MM}$ patients prior to melphalan therapy. Clinical data were also collected from these patients following melphalan therapy. Results: rs 4240803 was associated with elevated expression of SLC7A5 mRNA, higher ex vivo sensitivity to melphalan in PBMCs, and positive 90-day response in these patients ( $p=0.047,0.10,0.049$, respectively). Conclusion: rs 4240803
\end{abstract}

This article is freely accessible online.

\#Jasmine A. Johnson was an undergraduate student at College of Pharmacy, The Ohio State University.

Correspondence to: Ming J. Poi, Division of Pharmacy Practice and Science, College of Pharmacy, The Ohio State University, Columbus, OH, 43210, U.S.A. Tel: +1 6142926622, e-mail: poi.2@osu.edu; Junan Li, Division of Pharmacy Practice and Science, College of Pharmacy, The Ohio State University, Columbus, OH, 43210, U.S.A Tel: +16142921036,e-mail: li.225@osu.edu

Key Words: Multiple myeloma, melphalan, SLC7A5, clinical response. impacted the expression of SLC7A5, thus contributing to the clinical response of MM patients to melphalan therapy.

Multiple myeloma (MM) affects approximately 83,000 US citizens and over $50 \%$ of patients die within 5 years of diagnosis. Autologous hematopoietic stem cell transplants (AutoHSCT) in MM improves overall survival by 1 year on average (1-4) and remains the standard of care for fit MM patients despite the increasing availability of novel agents, such as proteasome inhibitors, immunomodulatory drugs, and monoclonal antibodies. However, the treatment outcome of autoHSCT varies considerably among MM patients, and about $20 \%$ of patients exhibit melphalan-resistant myeloma, as demonstrated by a progression-free survival (PFS) of less than 12 months, much shorter than the PFS of the majority of MM patients $(3,4)$. Recent studies indicate that a variety of mechanisms, such as the influx and efflux of melphalan into cells $(5,6)$, DNA repair of melphalan-induced damage $(7,8)$, and melphalan metabolism/elimination $(9,10)$, may influence the patient's response to melphalan.

SLC7A5 (also known as LAT1, L-type amino acid transporter 1) is a $\mathrm{Na}^{+}$and $\mathrm{pH}$-independent neutral amino acid transporter $(11,12)$. In association with SLC3A2 (also designated as CD98 or $4 \mathrm{~F} 2 \mathrm{hc}$, a small type II membrane glycoprotein), SLC7A5 forms a heterodimeric transmembrane protein complex that catalyzes the transport of large neutral amino acids, such as leucine, phenylalanine, tyrosine, and histidine $(11,13-16)$. It has been reported that melphalan, a bis(2-chloroethyl) amino derivative of Lphenylalanine, is recognized and transported into cells by SLC7A5 $(6,17,18)$. While down-regulation of SLC7A5 expression has been found to be associated with melphalan 
uptake in different MM cell lines (6), a single nucleotide polymorphism (SNP) in the first intron of SLC7A5 (rs4240803, a G $\rightarrow$ A transition) was associated with the need of total parenteral nutrition (TPN; an indicator of melphalan-caused mucosal injury) in a cohort of MM patients after autoHSCT therapy (9). These results suggest that SLC7A may influence the cellular intake of melphalan as well as the toxicity of melphalan in MM patients. This premise has been further supported by our recent pharmacokinetic (PK) modeling study showing that SLC7A5 rs4240803 might influence melphalan distribution within the peripheral compartment, thus contributing to the interpatient variability in melphalan PK properties (10). However, whether and how rs4240803 impacts the pharmacodynamics (PD) of melphalan, especially the efficacy and the toxicity in MM patients is unknown.

The aim of our study was to evaluate the potential correlation of rs4240803 with SLC7A5 expression and clinical outcomes, namely, 90-day response, progression-free survival (PFS), and the severity of oral mucositis in MM patients after autoHSCT.

\section{Materials and Methods}

Peripheral blood mononuclear cell (PBMC) samples. Prior to melphalan infusion, samples of venous blood were obtained from 108 MM patients who underwent melphalan-based autologous stem cell transplantation as part of a prospective clinical trial were procured following approval from the Cancer Institutional Research Board's guidance (NCT01653106) (10). PBMCs were separated using Ficoll-Hypaque (Sigma-Aldrich, St Louis, MO, USA) at The Ohio State University Leukemia Tissue Bank as previously described (10).

Genotyping and gene expression assays. DNA and RNA were purified from PBMCs using a Blood DNA/RNA Purification kit (Qiagen, Valencia, CA, USA). cDNAs were synthesized using a High Capacity cDNA Reverse Transcription kit (Life Technologies, Carlsbad, CA, USA). Genotypes of SLC7A5 rs4240803 were determined on a QuantStudio ${ }^{\mathrm{TM}} 7$ Flex system (Thermo Fisher Scientific, Waltham, MA, USA) using a pre-validated TaqMan genotyping assay: C_1228783_10 (Thermo Fisher Scientific). Assays were conducted in duplicates.

The mRNA expression levels of target genes in MM cells and PBMCs were quantitatively assessed using TaqMan gene expression assays (Life Technologies) using the following inventoried primer/probes: Hs01001189_m1 (solute carrier family 7 member 5, SLC7A5), Hs00374243_m1 (solute carrier family 3 member 2, SLC3A2); Hs99999905_m1 (glyceraldehyde 3phosphate dehydro-genase, GAPDH). GAPDH was used as an endogenous reference for normalized gene expression. Each gene was amplified separately, and all experiments were performed in triplicate. The relative expression level (REL) of a target was determined using a comparative $\mathrm{Cq}$ method in which REL was defined as $2^{-\delta C q}$ (19). Of note, the expression level of a target gene presented in this study should be regarded as the baseline expression level of that specific target gene in PBMCs prior to melphalan treatment.
Ex vivo cell viability assays. PBMCs were seeded at 5000 cells/well in 96-well plates in $100 \mu \mathrm{l}$ of complete medium containing Advanced RPMI1640 (Life Technologies Corporate) supplemented with 5\% fetal bovine serum (FBS; Life Technologies,) and $1 \times$ Antibiotic Antimycotic solution (Sigma-Aldrich, St Louis, MO, USA). Cells were grown at $37^{\circ} \mathrm{C}$ in a $90 \%$ humidified environment in $5 \% \mathrm{CO}_{2}$ overnight. Subsequently, cells were incubated in complete medium containing various concentrations of melphalan (Sigma-Aldrich, St Louis, MO, USA) for another $24 \mathrm{~h}$. Cell growth was assayed using a WST-1 Cell Proliferation Assay Kit (Roche, Indianapolis, IN, USA). Assays were performed in triplicate. Absolute $\mathrm{IC}_{50}$ values (the concentration of melphalan required to inhibit $50 \%$ of the cell viability) were determined using Kaleidagraph software (Synergy Software, Reading, PA, USA) as previously described (10).

Pharmacokinetic $(P K)$ parameter determination. Venous blood samples were collected in heparin tubes prior to melphalan administration (time 0 ) and then at designated time points following completion of melphalan infusion: 5, 30, 45, 60, 180, and $360 \mathrm{~min}$. The plasma concentration of melphalan was assessed using liquid chromatography-tandem mass spectrometry (LC-MS/MS) (10). Non-compartmental PK parameters, such as $\mathrm{AUC}_{\text {inf }}$, Cmax, Tmax, were determined using Phoenix WinNonlin (v6.3, Pharsight, Mountain View, CA, USA).

Statistical analyses. All statistical analyses were conducted using R3.3.2 (R Foundation for Statistical Computing; cran.r-project.org). For rs24240803, the consistency between its distribution and the Hardy Weinberg equilibrium (HWE) principle was analyzed using chi-square test. Count data were analyzed using Fisher's exact tests or chi-square tests.

Gene expression data were subjected to log transformation to fulfill the normality requirement. Group wise differences in gene expression were analyzed using unpaired two-sample Student's $t$-tests where appropriate. The correlations between SLC7A5 and SLC 3A2 gene expression, between $S L C 7 A 5$ gene expression and ex vivo $\mathrm{IC}_{50}$ values were evaluated and tested using Pearson's product-moment correlation coefficients (19).

Time to relapse was determined as the time from transplantation until the earliest of the following time points: progressive disease, clinical relapse, or relapse from CR (complete response) as determined by the International Myeloma Working Group (IMWG). Patients without known progression were censored at the date of last follow up. Time to relapse was calculated from Kaplan-Meier curves with the difference between the curves analyzed using the log-rank test.

The potential association between mucositis (no: mucositis grade 0 and 1; yes: mucositis grade 2 and 3 ) and SNP as well as other covariates, between response at day 90 after transplantation (poor response: negative, minor response and partial response; good response: very good partial response, complete response, and stringent complete response) and SNPs as well as other covariates were evaluated using logistic regression.

$p$-Values were two-sided, and unless specified, values of $p<0.05$ were regarded as statistically significant.

\section{Results}

SLC7A5 rs4240803 in PBMCs. The majority of patients were Caucasian $(95 / 108,88.0 \%)$, such that the minor allele frequency (MAF=0.296) is comparable with the previously 
Table I. Demographics and clinical characteristics of patients in the current study.

\begin{tabular}{cccc}
\hline Characteristics & SLC7A5 & SLC7A5 & $p$-Value** \\
& rs42420803 & rs42420803 & \\
& GG & AG/AA \\
$(\mathrm{n}=53)$ & $(\mathrm{n}=55)$ & \\
& &
\end{tabular}

\begin{tabular}{|c|c|c|c|}
\hline \multicolumn{4}{|l|}{ Age (years) } \\
\hline Median (range) & $60(35-70)$ & $59(37-72)$ & 0.89 \\
\hline Gender & & & 0.57 \\
\hline Female & $26(49.1 \%)$ & $23(41.8 \%)$ & \\
\hline Male & $27(50.9 \%)$ & $32(58.2 \%)$ & \\
\hline \multicolumn{4}{|l|}{ Race } \\
\hline White & $45(84.9 \%)$ & $50(90.9 \%)$ & 0.43 \\
\hline Other & $8(15.1 \%)$ & $5(9.1 \%)$ & \\
\hline Melphalan dose* & & & 0.23 \\
\hline $140 \mathrm{mg} / \mathrm{m}^{2}$ & $6(11.3 \%)$ & $12(21.8 \%)$ & \\
\hline $200 \mathrm{mg} / \mathrm{m}^{2}$ & $47(88.7 \%)$ & $43(78.2 \%)$ & \\
\hline $\mathrm{CrCL}(\mathrm{ml} / \mathrm{min})$ & & & 0.64 \\
\hline $\mathrm{CrCL}<60$ & $8(15.1 \%)$ & $11(20 \%)$ & \\
\hline $\mathrm{CrCL} \geq 60$ & $45(84.9 \%)$ & $\begin{array}{l}43(78.2 \%) \\
(1 \text { missing) }\end{array}$ & \\
\hline \multicolumn{4}{|l|}{$\operatorname{AUCinf}(\mathrm{mg} * \min / \mathrm{l})$} \\
\hline Median (range) & $878(336-1640)$ & $871.5(388-1580)$ & 0.92 \\
\hline Risk & & & 0.18 \\
\hline Standard risk & $35(66.0 \%)$ & $25(45.5 \%)$ & \\
\hline Intermediate/high risk & $\begin{array}{l}17(32.1 \%) \\
(1 \text { missing })\end{array}$ & $\begin{array}{l}27(49.1 \%) \\
(3 \text { missing) }\end{array}$ & \\
\hline Mucositis & & & 0.96 \\
\hline 0 & $19(35.8 \%)$ & $21(38.2 \%)$ & \\
\hline 1 & $25(47.2 \%)$ & $24(43.6 \%)$ & \\
\hline 2 & $8(15.1 \%)$ & $7(12.7 \%)$ & \\
\hline 3 & $1(1.9 \%)$ & $\begin{array}{c}2(3.6 \%) \\
(1 \text { missing })\end{array}$ & \\
\hline \multicolumn{4}{|l|}{ Response at Day 90} \\
\hline NE/MR/PR & $23(43.4 \%)$ & $14(25.5 \%)$ & \\
\hline VGPR/CR/sCR & $17(32.1 \%)$ & $24(43.6 \%)$ & \\
\hline & (13 missing) & (17 missing) & \\
\hline \multicolumn{4}{|l|}{$\begin{array}{l}\text { Length of stay in } \\
\text { hospital (days) }\end{array}$} \\
\hline Median (range) & $14(11-21)$ & $14(10-21)$ & 0.81 \\
\hline
\end{tabular}

Sd: Standard deviation; CrCL: creatinine clearance. *Melphalan dose adjusted by the patient's BSA (body surface area, $\mathrm{m}^{2}$ ). **All count data were analyzed using Chi square tests or Fisher's exact tests where appropriate, and continuous data were analyzed using $\mathrm{U}$ rank sum tests. All tests were two-sided. Sd: Standard deviation. Missing data were not included in the statistical analyses. ***For 90-day response, NE: Not evaluable; MR: minor response; PR: partial response; VGPR: very good partial response; CR: complete response; sCR: stringent complete response.

reported MAF (0.282) in a Caucasian population (9). The genotype frequencies of rs4240803 are in Hardy-Weinberg equilibrium $(p=0.99)$. A total of 53 patients carried the wild type genotype GG, 46 patients were heterozygous GA carriers; and 9 patients harbored the homozygous AA variant genotype. Table I summarizes the demographic and clinical characteristics of these patients. There is no statistically significant association between rs4240803 GG and GA/AA carriers with age, sex, race (white, other), risk (standard, intermediate/high), melphalan dose $\left(140 \mathrm{mg} / \mathrm{m}^{2}, 200 \mathrm{mg} / \mathrm{m}^{2}\right)$, creatinine clearance $(\mathrm{CrCL}<60 \mathrm{ml} / \mathrm{min}, \mathrm{CrCL} \geq 60 \mathrm{ml} / \mathrm{min}$ ), AUC (area under the concentration-time curve, $\mathrm{mg}^{*} \mathrm{~min} / \mathrm{l}$ ), length of stay, and oral mucositis score (all $p$-values $>0.15$ ).

Rs4240803 is located within the first intron of the SLC7A5 gene in the proximity of a putative transcription enhancer (9). It is anticipated that rs4240803 may impact the transcription of SLC7A5 rather than directly the SLC7A5 protein. To address this premise, we evaluated the mRNA expression of SLC7A5 in PBMCs from patients with different rs42420803 genotypes. As shown in Figure 1A, the expression level of SLC7A5 mRNA was significantly associated with rs42420803 genotype $(p=0.047)$. The expression level of SLC7A5 mRNA in patients with rs4240803 A* variant alleles (AG/AA) was higher than that in patients homozygous GG at rs4240803. Since SLC7A5 functions as a heterodimer with a covalently-bound SLC3A2 unit (through a disulfide bond) $(20,21)$, the expression level of SLC3A2 mRNA was also evaluated in these PBMC samples. Overall, there is a strong positive correlation between $S L C 7 A 5$ and $S L C 3 A 2$ in mRNA expression (Pearson's correlation coefficient $=0.46, p<0.001$ ) (Figure 1B). Since SLC7A5, in association with SLC3A2, functions in the uptake of melphalan in MM cells, we anticipated that elevated SLC7A5 expression in PBMCs from patients with rs4240803 AG/AA genotypes would enhance the uptake of melphalan and sensitize these cells. Results from our ex vivo cytotoxicity assays support this premise. As shown in Figure 1C, PBMCs with higher SLC7A5 mRNA expression tended to have lower $\mathrm{IC}_{50}$ values of melphalan $(p=0.10)$, indicating that these cells were more sensitive to melphalan. This observation was consistent with previous findings that as a melphalan transporter, downregulation of SLC7A5 expression by siRNA led to reduced intake of melphalan in MM cell lines (6).

SLC7A5 rs42420803 and clinical outcomes in MM patients. Subsequently, the potential association between rs4240803 and 90-day response in these MM patients was investigated. As shown in Table I, 63.1\% (24/39) of patients with 90-day response data and rs42420803 AA/AG had "good" 90-day responses (including very good partial response, complete response, and stringent complete response), considerably higher than the response rate $42.5 \%(p=0.07)$ of rs42420803 GG patients. Univariate logistical regression analyses showed that rs4240803, melphalan dose, and renal function (CrCL) were associated with 90-day response in these MM patients. The odds ratio (OR) of having 90-day response between rs4240803 AA/AG and GG was 2.32 (AA/AG vs. $\mathrm{GG}, 95 \% \mathrm{CI}=0.94-5.87, p=0.07)$; OR between the various melphalan dose levels was $3.49\left(200 \mathrm{mg} / \mathrm{m}^{2}\right.$ vs. $140 \mathrm{mg} / \mathrm{m}^{2}$, 95\% $\mathrm{CI}=0.92-17.0, p=0.08$ ); $\mathrm{OR}$ between the various $\mathrm{CrCL}$ 
A

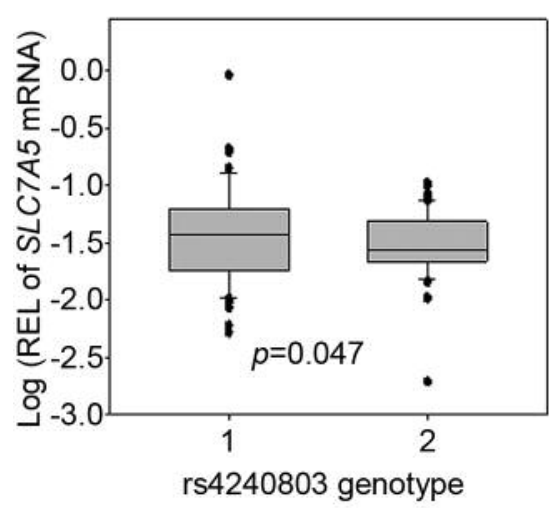

B

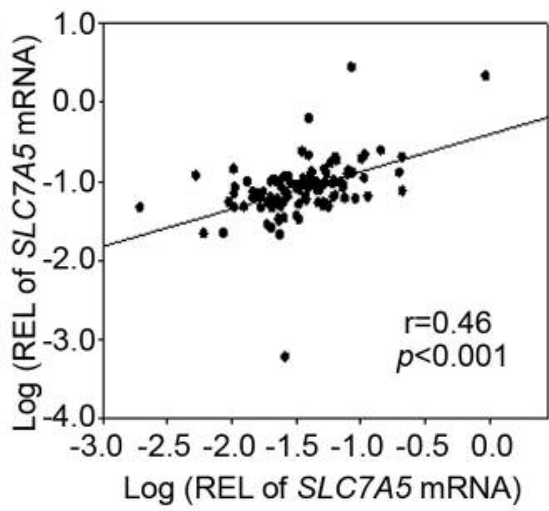

C

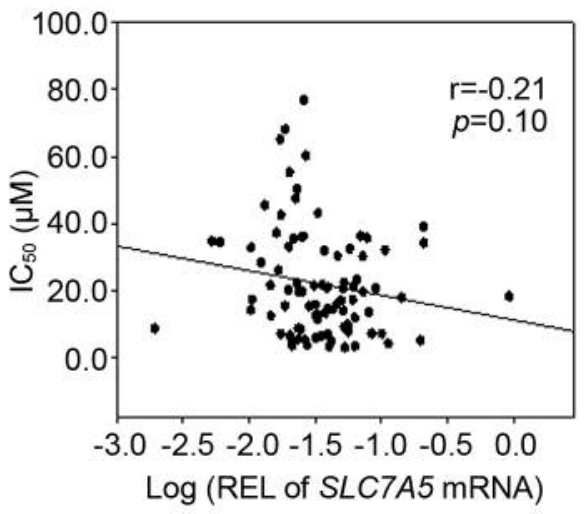

Figure 1. Association of SLC7A5 rs4240803 genotypes (AA/AG, GG) with gene expression and ex vivo sensitivity to melphalan in PBMCs from MM patients. A) Box plots to analyze the expression of SLC7A5 mRNA in PBMCs from MM patients with different SLC7A5 rs4240803 genotypes (AA/AG, GG). qRT-PCR-based assays using pre-validated primers and probes were used to determine the mRNA expression levels of target genes. GAPDH was used as an endogenous reference, and the relative expression level (REL) of a target gene was defined using the 2- $\Delta C q$ method (19). Each assay was conducted in triplicate and the average value was used for statistical analyses. All expression data were subjected to log transformation to fulfill the normality requirement, and two-sided Student's t-test was used to analyze the difference in SLC7A5 expression between patients with different rs42420803 genotypes: 1, AA/AG; 2, GG. B) Scatter plots illustrating the correlation between SLC7A5 and SLC3A2 in mRNA expression in PBMCs from MM patients. The potential correlations were analyzed using Pearson's correlation tests. $r$, Pearson's correlation coefficient. C) Scatter plots illustrating the correlation between SLC7A5 mRNA expression and IC ${ }_{50}$ values of melphalan in PBMCs from MM patients. IC ${ }_{50}$ values were evaluated using WST-1 cell proliferation assays as previously described. (10). Assays were performed in triplicate, and the average of three $I_{50}$ values was used in the statistical analyses.

levels $(<60 \mathrm{ml} / \mathrm{min}$ vs. $\geq 60 \mathrm{ml} / \mathrm{min}, 95 \% \mathrm{CI}=0.87-11.9$, $p=0.09$ ). In contrast, the association between 90-day response and age, sex, risk, $\mathrm{AUC}_{\mathrm{inf}}$ were insignificant $(p>0.2)$. With adjustments by melphalan dose and CrCL, OR between SLC7A5 rs4240803 AA/AG and GG was 2.70 (95\%CI=1.02-7.69, $p=0.049)$. SLC7A rs4240803 $(\mathrm{G} \rightarrow \mathrm{A})$ favored 90-day response in MM patients after autoHSCT. Further analyses revealed a marginally significant association between 90-day response and the expression level of SLC7A5 mRNA in the cohort of MM patients in our study $(p=0.15)$ and patients with higher SLC7A5 mRNA expression trended to have higher odds of "good" 90-day response. These results are consistent with a previous study showing that the overall response rate in MM patients with high SLC7A5 protein expression was significantly higher than that in patients with low SLC7A protein expression (60\% vs. $17.6 \%, p=0.03)(21)$.

Furthermore, we investigated the potential association of rs4240803 with PFS or the severity of oral mucositis. Our results did not show any significant association for either comparison $(p=0.66,0.97$, respectively). Interestingly, results from a previous study indicated that rs4240803 was significantly associated with TPN requirement in MM patients, a global measure of gastrointestinal toxicity (9). Compared with patients carrying rs4240803 GG, patients with rs4240803 A* alleles had lower odds of IPN use $(\mathrm{OR}=0.45,95 \% \mathrm{CI}=0.25-0.79, p=0.007)$, indicating that rs4240803 favored lower gastrointestinal toxicity. Notably, TPN requirement was used as an index of gastrointestinal toxicity encompassing clinical manifestations including pain, nausea, and diarrhea. Therefore, it served only as a proxy for chemotherapy-induced mucositis due to lack of formal mucositis scores for the majority of studied patients (9).

\section{Discussion}

SLC7A5 and SLC7A8 are L-type amino acid transporters involved in the passage of large neutral amino acids such as leucine, isoleucine, phenylalanine, tyrosine, tryptophan, methionine, histidine, and valine into the cell (5). While SLC7A8 is expressed primarily in the kidneys and bowel (22), SLC7A5 is widely expressed in all human tissues except intestine (13). As such, SLC7A5 is assumed to play a more important role than SLC7A8 in amino acid intake in dividing cells (6). Elevated expression of SLC7A5 has been found in a variety of human cancer cells and tumors, including lung, brain, prostate, stomach, and pancreatic cancers (21). It is generally believed that the expression levels of SLC7A5 are relevant to tumor development, angiogenesis, and poor prognosis in cancer patients $(9,21)$. In multiple myeloma, SLC7A5 and SLC7A8 are regarded as the major mediators of melphalan uptake in cells, due to the high resemblance between melphalan and phenylalanine in molecular structure and the binding affinity to SLC7A5 (5). 
While it has been found that melphalan competes with endogenous large neutral amino acids for SLC7A5 binding, transcriptional knockdown of SLC7A5 by siRNA led to decreased intake of melphalan in MM cells, indicative of the crucial roles of SCL7A5-mediated transport in the absorption and distribution of melphalan (6). Furthermore, Isoda et al. (21) reported that MM patients who have a higher SLC7A5 protein expression were more likely to have a positive clinical response after treatment with melphalan and prednisolone, implying that the functions of SLC7A5 could influence the clinical response in MM patients. In the present study, we quantitatively evaluated the impact of rs4240803 on the expression of SLC7A5 mRNA and the sensitivity of PBMCs to melphalan in $108 \mathrm{MM}$ patients with standard autoHSCT-HDM treatment. rs4240803 was significantly associated with increased SLC7A5 mRNA expression in PBMCs, resulting in greater sensitization of these cells to melphalan cytotoxicity. Moreover, rs4240803 was associated with a higher possibility to have a positive 90-days response in these patients. To our knowledge, this is the first report on the association of SLC7A5 rs4240803 with a positive clinical response in MM patients.

Our study has also provided novel insights into understanding the mechanisms underscoring the observed association between rs4240803 and clinical response. Even though up-regulation of SLC7A5 at mRNA and protein levels has been previously found in different human cancers, including multiple myeloma (21), little is known about the cis- and trans-regulatory factors that control transcription of SLC7A5. The proximity of rs42400803 to a putative enhancer segment in the first intron of SLC7A5 (9) provides a rationale for predicting a functional role in modifying transcription of SLC7A5. Our data showed that rs42400803 correlated with enhanced transcription of SLC7A5 in PBMCs. This is the first report on the molecular mechanisms underlying the impact of rs4240803 in MM patients. Because of the well-documented similarities in the molecular characteristics between PBMC and bone marrow plasma cells (BMPC) from the same patient, we anticipate that rs4240803 could up-regulate the transcription of SLC7A5 in BPMCs as well, subsequently increasing the uptake of melphalan into these tumor cells. Higher intake of melphalan would enhance the cytotoxicity of melphalan in BPMCs and consequently lead to better clinical response in MM patients carrying this variation.

It is important to note that multiple molecular mechanisms may be involved in the regulation of SLC7A5 at the transcriptional and post-transcriptional levels, which would ultimately influence the functioning of SLC7A5 in MM patients. First, it has been found that SLC7A5 is overexpressed in many human cancers with an increased demand of amino acids, suggesting that the expression of SLC7A5 might be inducible $(5,16)$. Second, there is a considerable number of SNPs identified in the coding and non-coding regions of SLC7A5 that may impact the transcription of $S L C 7 A 5$ individually or in combination (9, $10,18)$. While rs4240803 was found to be associated with the gastrointestinal toxicity and the distribution of melphalan in the peripheral compartment in two different cohorts of MM patients, respectively $(9,10)$, a group of 44 polymorphisms in SLC7A5 (excluding rs42420803) were identified in a previous study including $64 \mathrm{MM}$ patients (18). These 44 SNPs were located in coding/non-coding regions other than intron 1 , including 5 ' flank region, exon 1 , and introns 2-7, and none of them were found to be significantly associated with the expression of SLC7A5 mRNA. However, we cannot rule out the possibility that the impact of rs4240803 on SLC7A5 transcription is influenced by some of these additional SLC7A5 SNPs through linkage-equilibrium or other molecular mechanisms, i.e. some of these SNPs might have a bearing on SLC7A5 transcription in the presence of rs4240803. Third, it has been reported that different amino acids (as well as melphalan) compete with each other for binding to SLC7A5 in cells $(14,23,24)$. As such, SLC7A5-mediated transport of melphalan in cells could be impacted by certain amino acids (such as glutamine, histidine, leucine) and the action of amino acid transporters other than SLC7A5. Taken together, these observations suggest that the putative impacts of rs4240804 on SLC7A5 transcription and subsequent functioning in MM patients could be "cofounded" with effects caused by other SLC7A5-relevant or irrelevant molecular mechanisms/pathways. Arguably, rs4240803, like polymorphisms in most drug metabolismrelated genes, would exert a moderate or even "small" effect on melphalan metabolism and functioning in MM patients (25), hence, meta-analyses and more extensive analyses in large cohorts of MM patients in controlled clinical trials would be essential to elucidate the contribution(s) of this and other SLC7A5 SNPs to the interpatient heterogeneity of melphalan response. The relatively small size $(\mathrm{N}=108)$ of the patient population and the unavailability of clinical data (notably for 90-day response) from a considerable portion of patients limited the full testing of some of our hypotheses and conservatively restricted our ability to demonstrate definitive outcomes. Nonetheless, our results strongly support further studies to elucidate the potential roles of SLC7A5 rs4240803 (and SLC7A5 in general) in MM patients with autoHSCT-HDM therapy.

\section{Acknowledgements}

The Authors thank Dr. Thomas Knobloch, The Ohio State University, for critically reading the manuscript. This study was partly supported by a Pelotonia IDEA award (46050-502048; MAP, $\mathrm{CCH}, \mathrm{MJP})$ and a startup research Grant from the College of Pharmacy, The Ohio State University (MJP). 


\section{References}

1 Rajkumar SV and Kyle RA: Multiple myeloma: diagnosis and treatment. Mayo Clin Proc 80: 1371-1382, 2005.

2 Bayraktar UD, Bashir Q, Qazilbash M, Champlin RE and Ciurea SO: Fifty years of melphalan use in hematopoietic stem cell transplantation. Biol Blood Marrow Transplant 19: 344-356, 2013.

3 Shaw P, Nath C and Lazarus H: Not too little, not too much just right! (Better ways to give high dose melphalan). Bone Marrow Transplant 49: 1457-1465, 2014.

4 Gay F, Oliva S, Petrucci MT, Conticello C, Catalano L, Corradini P, Siniscalchi A, Magarotto V, Pour L, Carella A, Malfitano A, Petrò D, Evangelista A, Spada S, Pescosta N, Omedè P, Campbell P, Liberati AM, Offidani M, Ria R, Pulini S, Patriarca F, Hajek R, Spencer A, Boccadoro M and Palumbo A: Chemotherapy plus lenalidomide versus autologous transplantation, followed by lenalidomide plus prednisone versus lenalidomide maintenance, in patients with multiple myeloma: a randomized, multicenter, phase 3 trial. Lancet Oncol 16: 1617-1629, 2015.

5 del Amo EM, Urtti A and Yliperttula M: Pharmacokinetics role of L-type amino acid transporters LAT1 and LAT2. Eur J Pharm Sci 35: 161-174, 2008.

6 Kuhne A, Tzvetkov MV, Hagos Y, Lage H, Burckhardt G and Brockmoller J: Influx and efflux transport as determinants of melphalan cytotoxicity: resistance to melphalan in MDR1 overexpressing tumor cell lines. Biochem Pharmacol 78: 45-53, 2009.

7 Gkotzamanidou M, Sfikakis PP, Kyrtopoulos SA, Bamia C, Dimopoulos MA and Soulliotis VL: Chromatin structure, transcriptional activity and DNA repair efficiency affect the outcome of chemotherapy in multiple myeloma. British J Cancer 111: 1293-1304, 2014.

8 Nath C, Trotman J, Tiley C, Presgrave P, Joshua D, Kerridge I, Kwan YL, Gurney H, McLachalan AJ, Earl JW, Nivision-Smith I, Zeng L and Shaw PJ: High melphalan exposure is associated with improved overall survival in myeloma patients receiving high dose melphalan and autologous transplantation. Br J Clin Pharmacol 82: 149-159, 2016.

9 Giglia JL, White MJ, Hart AJ, Toro JJ, Freytes CO, Holt CC, Cai Y, Williams SM and Brandt SJ: A single nucleotide polymorphism in SLC7A5 is associated with gastrointestinal toxicity after high-dose melphalan and autologous stem cell transplantation for multiple myeloma. Biol Blood Marrow Transplant 20: 1014-1020, 2014.

10 Cho YK, Sborov DW, Lamprecht M, Li J, Wang J, Hade EM, Gao Y, Tackett K, Williams N, Benson DM, Efebera YA, Rosko AE, Devine SM, Poi M, Hofmeister CC and Phelps MA: Associations of high-dose melphalan pharmacokinetics and outcomes in the setting of a randomized cryotherapy trial. Clin Pharmacol Ther 102: 511-519, 2017.

11 Kannai Y, Segawa H, Miyamoto K, Takeda E and Endou H: Expression cloning and characterization of a transporter for large neutral amino acids activated by the heavy chain of 4F2 antigen (CD98). J Biol Chem 273: 23629-23632, 1998.

12 Yanagida O, Kanai Y, Chairoungdua A, Kim DK, Segawa H, Nii T, Cha SH, Matuso H, Fukushima J, Fukasawa Y, Tani Y, Taketani Y, Uchino H, Kim JY, Inatomi J, Okayasu I, Miyamoto K, Takeda E, Goya T and Endou H: Human L-type amino acid transporter 1 (LAT1): characterization of function and expression in tumor cell lines. Biochim Biophys Acta 1514: 291-302, 2001.
13 Prasad PD, Wang H, Huang W, Kekuda R, Rajan DP, Leibach FH and Ganapathy V: Human LAT1, a subunit of system L amino acid transporter: molecular cloning and transport function. Biochem Biophys Res Commun 255: 283-288, 1999.

14 Uchino H, Kanai Y, Kim DK, Wempe MF, Chairoungdua A, Morimoto E, Anders MW and Endou H: Transport of amino acid-related compounds mediated by L-type amino acid transporter 1 (LAT1): insights into the mechanisms of substrate recognition. Mol Pharmacol 61: 729-737, 2002.

15 Augustyn E, Finke K, Zur AA, Hansen L, Heeren N, Chien HC, Lin L, Giacomini KM, Colas C, Schlessinger A and Thomas AA: LAT-1 activity of meta-substituted phenylalanine and tyrosine analogs. J Bioorg Med Chem Lett 26: 2616-2621, 2016.

16 Napolitano L, Galluccio M, Scalise M, Parravicini C, Palazzolo L, Eberini I and Indiveri C: Novel insights into the transport mechanism of the human amino acid transporter LAT1 (SLC7A5). Probing critical residues for substrate translocation. Biochim Biophys Acta 1861: 727-736, 2017.

17 Samuels BL and Bitran JD. High-dose intravenous melphalan: a review. J Clin Oncol 13: 1786-1799, 1995.

18 Kuhne A, Kaiser R, Schirmer M, Heider U, Muhike S, Niere W, Overbeck T, Hohloch K, Trumper L, Sezer O and Bockmoller J: Genetic polymorphisms in the amino acid transporters LAT1 and LAT2 in relation to the pharmacokinetics and isde effects of melphalan. Pharmacogenetics and Genomics 17: 505-517, 2007.

19 Poi MJ, Li J, Sborov DW, VanGundy Z, Cho YK, Lamprecht M, Pichiorri F, Phelps MA and Hofmeister: Polymorphism in ANRIL is associated with relapse in patients with multiple myeloma after autologous stem cell transplant. Mol Carcinog 56: 1722-1732, 2017.

20 Verrey F and System L: heteromeric exchangers of large, neutral amino acids involved in directional transport. Pflugers Arch 445: 529-533, 2003.

21 Isoda A, Kaira K, Iwashina $M$, Oriuchi N, Tominaga $H$, Nagamori S, Kanai Y, Oyama T, Asao T, Matsumoto M and Sawamura M: Expression of L-type amino acid transporter 1 (LAT1) as a prognostic and therapeutic indicator in multiple myeloma. Cancer Sci 105: 1496-1502, 2014.

22 Rossier G, Meier C, Bauch C, Summa V, Sordat B, Verrey F and Kuhn LC: LAT2, a new basolateral 4F2hc/CD98-associated amino acid transporter of kidney and intestine. J Biol Chem 274: 34948-34954, 1999.

23 Morimoto E, Kanai Y, Kim DK, Chairoungdua A, Choi HW, Wempe MF, Anzai N and Endou H: Establishment and characterization of mammalian cell lines stably expressing human L-type amino acid transporters. J Pharmacol Sci 108: 505-516, 2008.

24 Napolitano L, Scalise M, Galluccio M, Pochini L, Albanese LM and Indiveri C: LAT1 is the transport competent unit of the LAT1/CD98 heterodimeric amino acid transporter. Int J Biochem Cell Biol 67: 25-33, 2015.

25 Ioannidis JP, Trikalinos TA, Ntzani EE and ContopouslosIoannidis DG: Genetic associations in large versus small studies: an empirical assessment. Lancet 361: 567-571, 2003.

Received November 29, 2018

Revised December 8, 2018

Accepted December 10, 2018 